\title{
Fototesti di viaggio: Absolutely nothing di Giorgio Vasta e Ramak Fazel
}

\author{
Federico Fastelli
}

Il mio contributo vuole essere un primo tentativo di riflessione teorica, spero non troppo frettoloso, attorno ad un problema molto complesso che riguarda la definizione di un genere ibrido e, per così dire, sospeso. Per il momento possiamo chiamarlo 'fototesto di viaggio'. Mi servirò, come strumento di verifica, e cartina di tornasole del discorso teorico, di Absolutely Nothing di Giorgio Vasta e del fotografo Ramak Fazel, esempio tra i migliori che conosco, almeno in ambito italiano e in tempi recenti.

Diciamo subito che l'espressione 'fototesto di viaggio' descrive un ircocervo: una spaventosa fusione tra due generi, anch'essi, a propria volta contraddistinti da uno statuto particolarmente precario, cioè il reportage di viaggio e il fototesto.

Il reportage di viaggio è, di norma, un racconto fattuale, un racconto che si regge e giustifica attraverso la coincidenza nominale di narratore e personaggio viaggiatore, come sappiamo da Lejeune (1975). Nella modernità, generalmente, ciò si declina nell'uso deliberato del cosiddetto patto autobiografico, poiché la coincidenza coinvolge, sempre nominalmente, anche la figura extratestuale dell'autore. La posizione del narratore determina, come è ovvio, anche il tempo della narrazione, che è sempre ulteriore: per parlare di un viaggio avvenuto, occorre che esso, o la parte di esso che si sta raccontando, si siano conclusi. L'ascendenza di queste caratteristiche, in verità, è antica, e coinvolge in sostanza ogni racconto di viaggio, reale o immaginario che sia. Già nel modello omerico, 2021 Author(s), CC BY 4.0 International, DOI 10.36253/978-88-5518-467-0.31, in Michela Graziani, Lapo Casetti, Salomé Vuelta García (edited by), Nel segno di Magellano tra terra e cielo. Il viaggio nelle arti umanistiche e scientifiche di lingua portoghese e di altre culture europee in un'ottica interculturale, (C) 2021 Author(s), content CC BY 4.0 International, metadata CC0 1.0 Universal, published by Firenze University Press (www.fupress.com), ISSN 2704-5919 (online), ISBN 978-88-5518-467-0 (PDF), DOI 10.36253/97888-5518-467-0 
come è noto, l'atto di enunciazione del viaggio di Odisseo presso i principi Feaci è ceduto dal narratore extradiegetico di primo grado e dall'aedo della corte, Demodoco, che funge da primo narratore intradiegetico, allo stesso Odisseo. Né il primo né il secondo aedo, fino a quel momento, avevano avuto difficoltà a raccontare le vicende della guerra di Troia o quelle dei pretendenti al regno di Itaca, che, del resto, erano ad entrambi, a livelli diversi del racconto, sostanza del dettato delle muse. Il motivo della cessione della parola a Odisseo, come ha spiegato egregiamente Pino Fasano, fa eccezione rispetto ai numerosi episodi di 'racconto nel racconto' presenti nell'Iliade e in gran parte dell'epica classica successiva. Di norma il narratore intradiegetico di secondo grado è, come Demodoco, un cantore, un professionista della narrazione che non ha una 'funzione informativa' e non riferisce infatti di 'cose viste' (Fasano 2005, 166). Spesso è cieco, a rimarcare la 'funzione memoriale', di monumentalizzazione di «eventi, conoscenze, valori, norme etiche» (Fasano 2005, 166) già note agli ascoltatori, che vengono perciò fissate nella memoria dalla 'parola ornata', che è dono divino, e divengono esperienza. La scelta di Odisseo come narratore ha tutt'altro mandato poiché è volta a rendere conto di una serie di contenuti sconosciuti e distanti dal senso comune. Contenuti, cioè, derivanti dall'eversione dell'esperienza, per esempio da mondi lontani, che dovranno apparire accettabili agli ascoltatori per mezzo dell'auctoritas di chi li ha vissuti e adesso li sa raccontare.

Se, nell'epica, non si pone mai un problema di credibilità del narratore, poiché gli eventi sono già comunque noti anche al narratore extradiegetico di primo grado, in quanto sostanza del dettato delle muse o trasmissione della tradizione orale e quindi dell'esperienza, pure l'autenticazione del racconto, siccome si tratta di viaggio e vengono valicati i limiti del senso comune, è necessaria. Questa credibilità di 'cose viste' è perciò trasferita dal piano della verosimiglianza (o della conoscenza collettiva) a quello della forma della narrazione (o alla retorica): il racconto è così giustificato poeticamente, e ciò autorizza l'eventuale finzione sia sul piano narrativo che su quello etico. Odisseo, infatti, non è soltanto il primo viaggiatore testimone della nostra tradizione, ma è anche, come dimostra col suo discorso, incantando gli ascoltatori, il più abile dei narratori.

Un problema di verosimiglianza è spesso stato posto, invece, fuori dall'epica, ed ha appassionato il lettore moderno più di quanto non avesse fatto con quello dei secoli precedenti. Dall'antichità al Seicento, l'inattendibilità che a noi contemporanei fa sembrare un resoconto di viaggio 'fantastico' è in larga parte dovuta alla sua incompatibilità con ciò che chiamiamo il 'quotidiano' e dunque con il senso comune che - ha spiegato Giorgio Agamben - era, fino all'affermarsi della scienza moderna, il vero soggetto di una facoltà premoderna scomparsa, che tuttavia continuiamo a chiamare esperienza. Lautenticazione del racconto di viaggio poggiava allora saldamente sulla testimonianza e sull'autorità (appunto sulla reputazione e sull'abilità retorica del narratore), e ciò era sufficiente a ricondurre vicende, personaggi e luoghi incredibili in una dimensione di realtà, come ribadisce in maniera paradossale e antifrastica il Gulliver di Swift. Alla fine del racconto dei suoi viaggi, come si sa, l'avventuroso esploratore ci spiega che avrebbe potuto meravigliare i lettori con racconti assurdi e incredibili, e 
che invece si è limitato a riportare i fatti così come lui li ha visti, con l'intento di istruire più che di dilettare. E aggiunge di essere un seguace ligio del motto virgiliano nec si miserum Fortuna sinonem finxit, vanum etiam, mendacemque improba finget. L'irrisione del genere della letteratura di viaggio passa in Swift attraverso la messa in scacco, ed anzi al ribaltamento grottesco, proprio, dei consueti strumenti autenticativi legati all'onestà della testimonianza e all'auctoritas. Ma lo scetticismo dello scrittore si pone giusto a cavallo di quella frattura epocale che separa due mondi, due concezioni della realtà, e quindi anche due determinazioni di cosa sia vero e cosa sia falso. Da una parte il nascente mondo moderno, caratterizzato da un 'vincolo di realtà debole', come lo definisce Rino Genovese $(2008,29)$, sottoposto alla misurazione dei dati di fatto, e alla loro verifica, con conseguente esproprio dell'esperienza come facoltà intermedia tra l'intelletto (sovraindividuale) e i sensi. Dall'altra parte il mondo arcaico, antico e medioevale, in cui il vincolo di realtà è forte, e rispetto al quale la verità non viene mai posta come problema. Ecco perché non si può concordare, per esempio, con quanto afferma un grande scrittore come García Márques a proposito del racconto di Antonio Pigafetta del suo Primo viaggio attorno al mondo (1525) con il Magellano che qui si celebra: non c'è niente di fantastico in quel resoconto, se con fantastico intendiamo qualcosa, in senso moderno, di fuori dal dominio della realtà, $o$ anche se volessimo intendere, con armamentari teorici più raffinati, qualcosa di indecidibile rispetto al piano della realtà (cfr. Todorov 1970).

Dico questo, perdonerete la digressione genealogica, non solo perché nel reportage moderno si conserva, ed anzi si radicalizza la necessità di credibilità della narrazione - per cui il patto autobiografico, appunto, è il primo strumento retorico di ancoraggio dei contenuti alla fattualità -, ma anche perché, come vedremo, l'opera di Vasta e Fazel insiste molto sull'ambiguità delle categorie, spesso confuse tra loro, di falso e finto, così come su quelle di vero e di reale.

La fattualità del reportage moderno origina proprio dalla necessità di una costruzione, se così posso dire, di verità giustificata del narrato, e dunque come espressione di un vincolo di realtà particolarmente debole. Il reportage giornalistico nasce infatti come dispositivo formale di approfondimento di notizie già parzialmente note: il lettore è trasportato all'interno della news attraverso strumenti retorico-letterari che, di fatto, costruiscono, con la narrazione, un mondo, che è sempre in qualche modo una rappresentazione del mondo reale, è sempre un eterocosmo. Inoltre, si può dire con ferma convinzione, anche per relativizzare l'idea del tutto imprecisa secondo cui le cosiddette fake news sarebbero appannaggio assoluto della nostra epoca, che il reportage giornalistico nasce proprio dall'incontro tra news e fiction, ed in particolare laddove la fiction estende il proprio dominio sulle news. Avviene, in questi casi, che la scrittura giornalistica superi la mera refertazione (sulla cui fattualità, in ogni caso, si potrebbe discutere) della fabula dei fatti, per parafrasare il titolo di un importante studio di John Hellmann (1981), a vantaggio di una restituzione meno epidermica delle atmosfere, delle sensazioni e delle emozioni in cui quei fatti si sono realmente intrecciati. È inevitabile, allora, che questo tipo di scrittura si ponga a ridosso della letteratura, e mutui perciò dal campo della narrazione, e nella 
modernità soprattutto della narrazione romanzesca che è certamente egemone, non soltanto le tecniche, ma di fatto le strutture, i luoghi comuni e le consuetudini rappresentative. Potrebbe sembrare curioso che qualcosa che nasce per esigenze veridittive si serva di strumenti collaudati in ambito finzionale. Il punto, non lo si ripeterà mai abbastanza, è proprio che - come Lacan sapeva bene - la verità ha sempre la struttura di una finzione. E, d'altro canto, potremmo anche dire che la menzogna appartiene al reale tanto quanto il vero.

In senso molto generale, per quel che qui ci riguarda, basta sapere che spettacolarizzazione, deformazione, invenzione, strumentalizzazione sono pratiche vecchie quanto l'informazione stessa, e di certo non riguardano esclusivamente l'epoca di internet. Tutt'al più, ciò che varia, è la dimensione della loro pervasività. Già nei primi anni Sessanta, per esempio, si poteva teorizzare l'esistenza di pseudo-eventi creati per e dai media contemporanei, la cui attività, come spiegava allora Daniel Boorstin (cfr. 1962), aveva un peso sulla realtà, ben al di là del proprio valore di verità: il falso, lo ripeto, fa parte del reale tanto quanto il vero. E inoltre, come suggeriva Lippmann nel suo indimenticato Public Opinion (1922), non possiamo mai scordare che le news si occupano dei fatti, mentre la verità è sempre precisamente ciò che sta dietro quei fatti.

Ora, tutto ciò vale, come si capisce, anche per il reportage di viaggio, nonostante la genesi dell'odeporica preceda senza alcun dubbio la storia del giornalismo moderno, e il resoconto di itinerari realmente compiuti possa riportarci indietro, addirittura a Erodoto, e cioè a quando, come scrive ancora Fasano, per le strade dell'Asia, questi cerca di capire meglio le cause delle guerre persiane (cfr. Fasano 2005). I problemi formali del racconto fattuale sono identici, almeno nella modernità che segue la svolta fisiologico-filosofica, se posso usare questa brutta espressione, della scienza moderna. Nel corso degli ultimi due secoli, in particolare, così come il cronista-reporter utilizza strumenti veridittivi con funzione probatoria di una certa notizia o dimostrativa delle diverse opinioni su di essa, il viaggiatore è chiamato a giustificare l'effettività del proprio itinerario rispetto ai luoghi e alle persone che incontra, nel tentativo di smentire quel luogo comune nato proprio nel corso del Seicento che vuole ogni narratore di viaggio assimilabile al più vanaglorioso dei bugiardi: l'abilità retorica e l'autorità del proprio nome non sono più sufficienti. Uno di questi strumenti, peraltro sempre più spesso adoperati come generatori di effetti di realtà in opere di ordine biografico, autobiografico o anche autofinzionale, è la fotografia. Dalla metà dell'Ottocento in poi, e in particolare nel corso del Novecento, l'inclusione di fotografie all'interno della struttura del resoconto trasforma la natura di quest'ultimo in un fototesto.

Prendendo spunto dagli essenziali studi di Michele Cometa sull'argomento (2011 e 2016), possiamo chiamare fototesto una tipologia particolare di iconotesto, un prodotto cioè che mette in scena su di un unico supporto mediale testo e immagine, proponendo in apparenza l'utopica fusione dei due media, ma in realtà, come sappiamo dagli studi soprattutto di W. J. T. Mitchell, mostrando alla fine l'irriducibilità della loro differenza, ovverosia indicando «lo spazio di uno scarto tra verbale e visuale» (Cometa 2016, 73). La specificità del foto- 
testo rispetto all'iconotesto sta, ovviamente, nella sua componente fotografica, le cui caratteristiche peculiari (riproducibilità, referenzialità, indessicalità) ne stabiliscono funzioni, retoriche e finalità affatto tipiche. Da una prospettiva di studi di cultura visuale, la retorica del fototesto risulta in particolare dalla specificazione e dalla successiva reintegrazione di quelle che Cometa chiama retoriche dello 'sguardo', delle retoriche del layout, dei supporti e dei 'parerga'. In questo modo, e riassumendo all'osso il discorso, si possono distinguere almeno tre macro-forme di fototesto. Con forma-emblema si può intendere un fototesto che cerca di produrre effetti di lettura programmati, ancorché non univoci. Tale forma è da riconnettere al modello barocco che prevede la giustapposizione tra una inscriptio (un titolo), una pictura (la foto stessa) e una subscriptio (un epigramma, sentenza o commento che si riferisce in maniera più o meno criptica ed enigmatica ai primi due elementi). Bertold Brecht, per fare un esempio assai conosciuto, ha espresso le potenzialità di questa costruzione nel suo Kriegsfiebel, appunto 'l'abicì (delle immagini) della guerra'. Con forma-atlante, si può invece intendere un fototesto a significazione diffusa. In esso la costruzione ad album, priva di riguardo per la cronologia e per ogni altro ordine dichiarato, tende a disporre sulla pagina materiali eterogenei. Questa forma, la cui potenzialità acronica è stata sfruttata appieno dai pathosformel di Aby Warburg, per esempio, arriva fino alla declinazione estrema rappresentata dal collage di ascendenza avanguardistica. Infine, con forma-illustrazione possiamo intendere la forma più semplice di fototesto, e si tratta cioè della visualizzazione fotografica di un testo, ovverosia dell'azione speculare alla narrativizzazione di un'immagine, tradizionalmente costituita dall'ékphrasis. Se l'ékphrasis traduce intersemioticamente, come recita un'antica e un po' abusata formula di Jakobson, un'immagine in forma verbale, l'illustrazione compie il percorso inverso, traducendo una forma verbale in immagine.

In Absolutely Nothing dobbiamo considerare un doppio livello di fototestualità. Il primo funziona come forma-illustrazione: il racconto fattuale di Giorgio Vasta, infatti, è inframezzato e interrotto da fotografie scattate da Ramak Fazel, da sé stesso e da Silva, alias Giovanna Silva, fotografa ed editrice della collana Humboldt di Quodlibet, nonché promotrice dell'affascinante viaggio dei tre nel deserto americano, tra California, Arizona, Nevada, New Mexico, Texas e Louisiana. Questo primo livello è perfettamente coerente se prendiamo per buone le indicazioni testuali secondo cui, inizialmente, il viaggio avrebbe dovuto dar luogo ad una vera e propria guida turistica, tra ghost town, musei e scenari naturali di opere cinematografiche e videoclip. Come ha giustamente notato Niccolò Scaffai in una recensione uscita su «Alias» nell'ottobre del 2016, la particolarità più evidente del libro è proprio quella per cui «la narrazione mette in scena la trasformazione della guida di viaggio in romanzo. Mentre leggiamo, infatti, la vicenda soggettiva del protagonista prevale sull' illustrazione oggettiva del contesto» (Scaffai 2016, [s.p.]). Anche l'interazione delle fotografie con il racconto accompagna allora la stessa modificazione: l'apparente funzione illustrativa che le immagini avrebbero avuto se avessero potuto trovar posto in una vera guida di viaggio scolora di fronte all'incertezza referenziale del testo di Vasta. La fat- 
tualità del racconto resta appesa soltanto al meccanismo retorico della narrazione autobiografica, ma di fatto la struttura fototestuale manomette la verifica fattuale e promuove piuttosto la dimensione spaziale di un eterocosmo abitato da revenant. La fattualità diviene il pretesto per una riflessione complessiva sulla finzione, mentre le immagini, raffigurando spazi senza soggetti, si aprono ad una dimensione a propria volta fantasmatica. I pochi esseri umani immortalati non sono riconoscibili: il loro volto è sempre coperto, oppure è troppo distante. A volte sono colti di spalle e ciò amplifica un effetto di vuoto, di smarrimento e anche di straniamento, che gli ambienti del deserto e dell'archeologia industriale visitata dai tre protagonisti creano già di per sé. Lo stesso Vasta sente il bisogno di specificare questo aspetto. In uno dei molti commenti al racconto, presenti nel testo in corpo minore, scrive:

Ramak Fazel è il fotografo che ha scattato le foto di questo libro. È una persona. E questa dovrebbe essere - doveva essere - una guida di viaggio: narrativa, letteraria, ma una scrittura anche di servizio che traccia itinerari e fornisce informazioni mantenendo chi ha compiuto il viaggio se non sullo sfondo almeno in secondo piano. Solo che nel momento in cui alle otto di mattina del 2 ottobre 2013 Ramak Fazel compare nel cortile interno del Beverly Laurel Hotel di Los Angeles [...] l'asse di questo libro si modifica. Le persone si fanno personaggi, la tortuosità si innalza a metodo e la carrozza [del baedeker] si trasforma nella zucca di una scrittura che soprattutto suppone, finge, si arrangia, mente (Vasta, e Fazel 2016, 21).

Absolutely nothing si fa allora interrogazione generale ed esistenziale del suo protagonista narratore sul vero e sul falso, sull'aderenza delle parole al loro significato, sull'ordine e sul caos, sulla presenza e sull'assenza. Come ha notato anche Daniele Giglioli (2016), in questo contesto Giovanna Silva impersona l'ordine, col suo metodo che «salda il mondo - in apparenza chiaro, in realtà labile - alle parole, che nel descriverlo lo sostengono e lo proteggono» (Vasta, e Fazel 2016, 26), mentre Ramak Fazel rappresenta il Caos cercando «ogni momento di deviare, di scantonare, di sprecare tempo, convinto evidentemente che dovunque, e vieppiù nel deserto, sia impossibile perdersi come ritrovarsi» (Giglioli 2016, [s.p.]).

Gli Stati Uniti, in generale, e in particolare luoghi visitati dai tre viaggiatori, luoghi nei quali alcuni microscopici aggregati urbani si palesano come il frutto deteriorato di uno stesso meccanismo economico, che li ha prima creati, alimentati, resi appetibili, e poi abbandonati dopo la crisi del prezzo dell'argento, costituiscono di certo lo spazio privilegiato per questa interrogazione: la nostra pre-conoscenza e il nostro pre-giudizio, che mettono in moto i meccanismi di tutta la letteratura odeporica, dipendono, in un modo o nell'altro, da una loro vita virtuale, fantasmatica, mediale. Sono prima di tutto luoghi del nostro immaginario, nutrito di cinema, di musica, di letteratura, di videoclip, e solo dopo, in seconda battuta, sono luoghi reali. Luoghi, insomma, di una peculiare dimensione dell'inesperienza: benché esistano soprattutto in una misura, seconda, virtuale, tutti ne abbiamo una qualche conoscenza, anche noi che li guardiamo 
dalla specola marginale della vecchia Europa, perché, per quanto remoti possano essere, sono parte del centro del nostro polisistema culturale: rock star, comici, star di Hollywood vi hanno posato, anche se solo una volta e per poche ore, il loro celebre piede, magari per girare un film, un videoclip o incidere un album. Noi continuiamo a vederli e rivederli, sentirli e riascoltarli, ripetuti all'infinito, sebbene la loro reale condizione sia quella di luoghi abbandonati, che ospitano soltanto esistenze ormai sfigurate e postreme.

Si assiste in questo modo all'inversione del rapporto tra fiction e non-fiction, e ciò complica notevolmente il nostro già precario intendimento su cosa sia la verità e cosa la menzogna. Ci interessa di più, tuttavia, che la nostra preconoscenza di quei deserti, oltre ad avere un fortissimo mandato allegorico di primo livello, per così dire, sia anche in grado di modificare l'interplay tra testo e immagine, agendo a livello metadiscorsivo: se nella guida di viaggio tradizionale l'illustrazione fotografica sfrutta le caratteristiche di referenzialità e indessicalità della fotografia, per mostrare ciò che il testo descrive e per autenticarlo, qui avviene piuttosto il fenomeno contrario, e non può non venire in mente l'influenza evidentemente giocata sull'opera di Vasta dal modello costituito da uno scrittore come Sebald (cfr. Scaffai 2016). Intendo dire che, in maniera non dissimile da quella di romanzi eccezionali come Austerlitz o Gli anelli di Saturno, si assiste qui ad un trattamento doppiamente allegorico della fotografia, che diventa anche, a livello metanarrativo, il residuo di un impossibile riconoscimento autobiografico, brandello di una memoria perduta, frammento di un'assenza che il testo tenta faticosamente di ricucire. Ciò si spiega anche a partire dalla constatazione che Vasta fa del deserto americano quando lo descrive come l'immagine di un altro deserto, un deserto privato e personale, quello di una perdita irreparabile: «absolutely nothing è in realtà absolutely nobody» (Vasta, e Fazel 2016, 232). Come è stato notato da numerosi critici, e come del resto l'autore lascia intendere in più luoghi del testo, «l'oggetto di queste pagine è la sparizione di una persona» (Vasta, e Fazel 2016, 232), di una ragazza, e di una storia d'amore interrotta. Avviene perciò che il livello più basso della narrazione, quello letterale, sia appannaggio dell'immaginazione, laddove alla realtà tocca occupare una dimensione seconda, allusa, che abita imperfettamente, e con fatica, la prima: il deserto come luogo di disappropriazione, di sottrazione radicale, che scioglie i legami, e riporta la solitudine allo smarrimento. Un luogo vuoto che incarna l'esigenza umana del riempimento: un Reale che esige di diventare Realtà, per mezzo dell'attribuzione di senso che solo il soggetto può esercitare. Si capisce così la visione finale di una nube nel deserto, da cui escono cinque figure arrivate lì per divorare i tre protagonisti. La scena, che, come giustamente rileva Scaffai, ossessiona ma, allo stesso tempo attrae il narratore, è evidentemente ispirata dal motivo della famiglia antropofaga, ricorrente nell'immaginario americano. Al di là del riferimento preciso, il punto è che attraverso questa immagine la perdita si rovescia nuovamente nel desiderio di appropriazione, nella pulsione e allo stesso tempo nella repulsione di essere divorati. Nel corso degli circa 8000 chilometri percorsi, fatti di 
strade, autostrade statali highway interstate, le cittadine e poi le praterie i deserti le lande meravigliosamente vuote, lo splendore dell'absolutely nothing, miliardi di millimetri e di millisecondi, milligrammi di ossigeno penetrato nei corpi ed espulso degradato in anidride carbonica, miriadi di diottrie consumate nello sguardo, molecole d'aria disciolte nelle coane e convertite da qualche parte nell'archeonecefalo in memoria sensoriale, ma soprattutto lo spazio, la fatica e l'orgoglio dello spazio (Vasta, e Fazel 2016, 167),

l'immaginazione viene in un certo senso aggredita dall'immaginario. I luoghi di quello scenario non possono semplicemente venire restituiti alla fine del viaggio, come di norma succede nella letteratura odeporica, ma costituiscono in qualche modo anche l'inizio concettuale del viaggio stesso, la condizione fantasmatica della sua stessa possibilità e della sua natura, che è sì la perdita dell'io di Giorgio Vasta, ma che più in generale è l'alienazione del viaggiatore-consumatore contemporaneo. In un impeto di lucidità e disperazione questi può sognare di essere consumato a propria volta, ma è poco altro che un'allucinazione, un $\mathrm{mi}$ raggio del deserto merceologico che abitiamo.

Dicevo all'inizio di questa riflessione sulla costruzione fototestuale dell'opera, che esiste un secondo livello di fototestualità. Questo secondo livello è innescato dall'appendice al racconto, e si spiega soltanto alla luce del cortocircuito tra immaginario e immaginazione, ovvero alla constatazione della coincidenza del vero e del falso, del reale e del finzionale, di una realtà cioè come messa in posa, che è sì fattuale e concreta, ma allo stesso tempo è finta e posticcia, come una vita che in un certo senso ripete un copione, rivive qualcosa di preordinato, come quando si riavvolge una pellicola che si è appena visto, oppure quando si impersona una maschera di teatro. Mi riferisco all'album fotografico che chiude il volume, intitolato Corneal Abrasion, cioè letteralmente abrasione della cornea, a firma del solo Fazel. Le foto, questa volta a colori, recuperano alcuni dei momenti vissuti dai viaggiatori e alcuni dei personaggi incontrati. Ciò che letteralmente ha colpito e ferito l'occhio di Fazel viene così rimescolato e riattiva a posteriori la memoria, che è la sola facoltà capace di rendere il viaggio compiuto come esperienza cruciale dell'esistenza umana. La distanza dal testo e la separazione anche grafica dal racconto svincolano però l'immagine da ogni funzione meramente illustrativa, ne affrancano l'enorme potenzialità semantica dall'incatenante azione di ancoraggio che nella prima parte la componente verbale gli imponeva, e ne concedono al lettore un' interpretazione libera in forma d'atlante. La verità ricostruita dalla memoria non ha qui la forma rassicurante della finzione del racconto, ma quella alogica, irrazionale, tanto seducente quanto pericolosa dell'immagine. Le fotografie, effettivamente, si affiancano, emergendo dal bianco dello sfondo della doppia pagina, quattro alla volta. La disposizione cronologicamente inesatta rispetto all'intreccio narrato da Vasta, a sua volta fitto di incongruenze temporali di ordine e durata (soprattutto anacronie), crea collegamenti inediti, fa saltare il continuum ordinato di qualsiasi possibile fabula, fa stridere un ambiente con uno completamente differente, un personaggio con un altro. L'ambiguità degli scatti, che sembrano il prodotto di un'indessicalità 
falsa, costruita, congegnata a tavolino, consegna al fruitore frammenti di tempo cristallizzato assolutamente indecidibili: finti in maniera così perfetta da sembrare veri, ma anche veri in maniera così perfetta da sembrare finti. Si accetta qui, ormai, che il falso non si opponga al vero, che, come si legge nel racconto a proposito della piccola città di Calico (Vasta, e Fazel, 104), tra i due termini non ci sia più alcun conflitto. E si lascia il lettore-interprete nell'impossibilità di riconoscere nei personaggi in scena, quelli immortalati dalla scrittura di luce di Fazel, le persone reali che effettivamente li interpretano, come se fossimo di fronte ad uno spettacolo dell'immenso Andy Kauffman, più volte evocato dal testo, e, al cospetto della sua capacità di cancellare la soglia dello spettacolo, la separazione tra vita interpretata e vita interpretante, «venisse fuori un senso di inadeguatezza così forte da rendere inequivocabile che non avere idea di cosa fare sia la struttura costitutiva dell'umano» (Vasta, e Fazel, 86).

In nessun altro genere che nel fototesto di viaggio, quindi, la disperata necessità di autenticazione del vissuto esperienziale attraverso il racconto è sintomo della sua oggettiva impossibilità. L'opera di Vasta e Fazel ha questo enorme merito: autotematizzare in maniera decisamente problematica ciò che nella contemporaneità il racconto di viaggio e la fotografia tendono per prassi ad espungere da sé, ovvero il loro carattere rappresentativo. La fattualità del patto autobiografico, la fattualità della luce che immortala lo spazio e intrappola il tempo, sono accidenti, inezie, rispetto al mandato di verità dell'uomo che si rappresenta. Piuttosto che autenticarsi l'uno con l'altro, il racconto e le immagini lasciano emergere le faglie di quel continuum spazio-temporale che fa, per così dire, apparire reale la realtà. Mostrano la costruzione operata dall'immaginazione e dalla memoria, lasciano intendere, l'uno resistendo al fascino dell' istante, le altre opponendosi al potere razionalizzante del significato, che la finzione è ciò che struttura per tutti il reale, facendone qualcosa di sopportabile, di umano. Così facendo associano la struttura del volume alla struttura del mondo, ricordandoci che l'espropriazione dell'esperienza significa per l'uomo moderno la necessità dolorosa di relegare ogni dimensione fantasmatica in un altrove, nella follia, nell'allucinazione o nell' infanzia, per esempio. Ma anche che quella stessa dimensione fantasmatica - e non la sua misurabilità o la sua verificabilità - tiene letteralmente insieme i contorni della realtà in una sorta continuum spazio-temporale e verbo-audio-visivo talmente finto da sembrare vero e talmente vero da sembrare, almeno qualche volta, finto.

\section{Riferimenti bibliografici}

Boorstin, D.J. 1962. The Image. A Guide to Pseudo-Events in America. New York: Atheneum.

Cometa, M. 2011. "Fototesti. Per una tipologia dell'iconotesto in letteratura." In $\mathrm{La}$ fotografia. Oggetto teorico e pratica sociale, Atti del XXXVIII Congresso AISS. Relazioni, a cura di Vincenza Del Marcio, e Isabella Pezzini, 63-101. Roma: Edizioni Nuova Cultura.

Cometa, M. 2016. “Forme e retoriche del fototesto letterario." In Fototesti. Letteratura e cultura visuale, a cura di Michele Cometa, e Roberta Coglitore, 69-115. Macerata: Quodlibet. 
Fasano, P. 2005. Letteratura e viaggio. Roma-Bari: Laterza.

Genovese, R. 2008. Gli attrezzi del filosofo. Difesa del relativismo e altre incursioni. Roma: Manifestolibri.

Giglioli, D. 2016. “Dove il passato è un futuro dimenticato.” La Lettura - Corriere della Sera, 11 settembre, 2016.

Hellmann, J. 1981. Fables of Fact. The New Journalism as New Fiction. Chicago: University of Illinois Press.

Lejeune, P. 1975. Le Pacte autobiographique. Paris: Seuil.

Lippmann, W. 1922. Public opinion. New York: Harcourt, Brace and Company.

Scaffai, N. 2016. "Spazio, metonimia di una mancanza." Alias - il manifesto, 16 ottobre, 2016.

Todorov, T. 1970. Introduction à la littérature fantastique. Paris: Seuil.

Vasta, G., e R. Fazel. 2016. Absolutely Nothing. Storie e sparizioni nei deserti americani. Macerata: Quodlibet. 